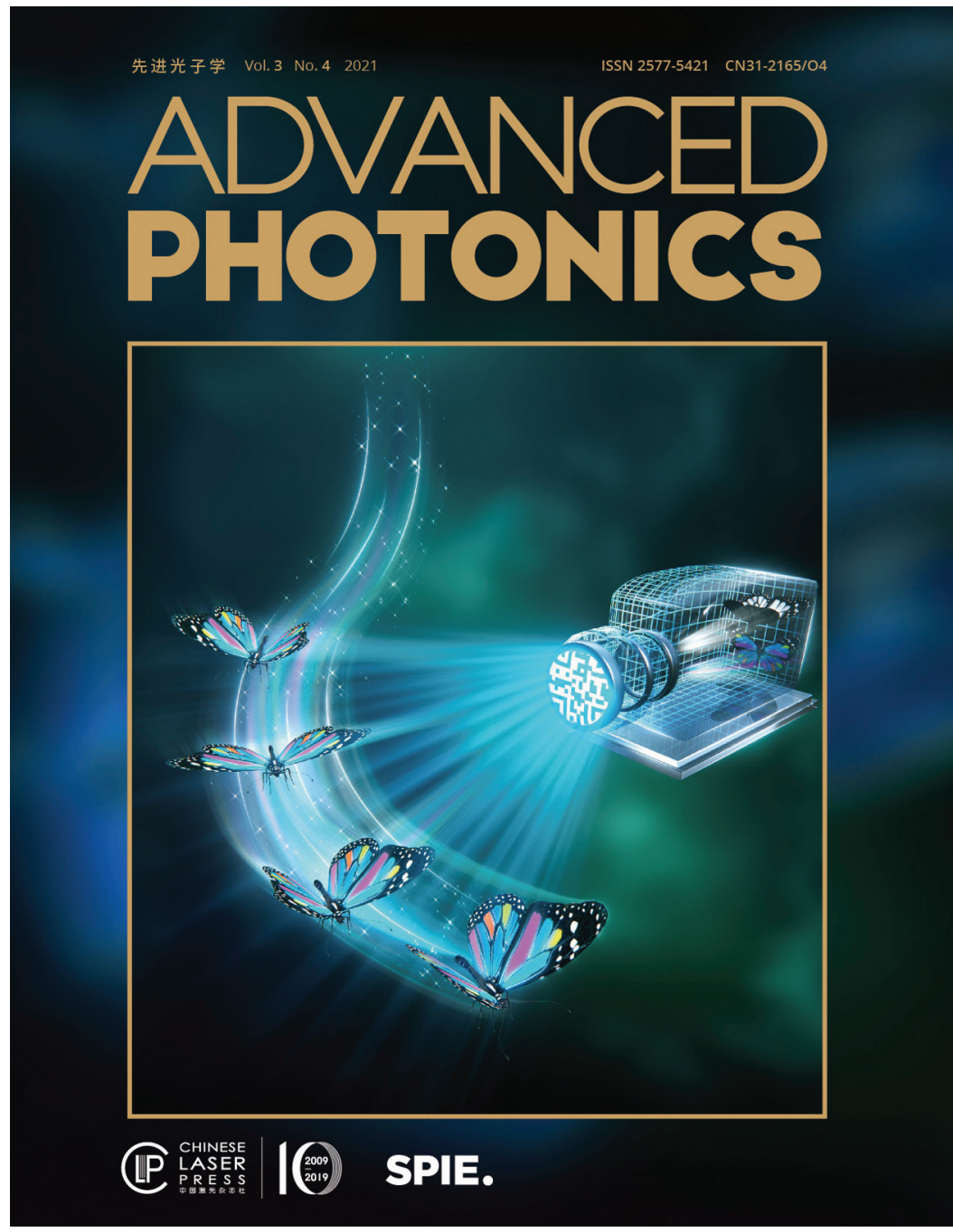

\title{
About the cover: Advanced Photonics Volume 3, Issue 4
}

The image on the cover for Advanced Photonics Volume 3 Issue 4 depicts a fast-flying butterfly with colorful information on its wings - this dynamic scene is captured by a multidimensional computational camera with super high temporal, volumetric, and spectral resolution. The camera is built based on compressed ultrafast imaging technology, which can simultaneously capture 5D information in a single snapshot by combining compressive sensing with temporal-to-spatial and spectral-tospatial conversion techniques.

The image is based on original research from East China Normal University State Key Laboratory of Precision Spectroscopy, as presented in the report by Pengpeng Ding, Yunhua Yao, et al., "Single-shot spectral-volumetric compressed ultrafast photography," Adv. Photonics 3(4), 045001 (2021). 